\title{
Steiner Tree: approach applying for shortest path in selected network
}

\author{
Manisha Wadhwa, Dr.Kanak Saxena \\ Department of Computer Applications \\ Samrat Ashok Technological Institute, Vidisha M.P. India
}

\begin{abstract}
This paper focus on approach for shortest path from source to destination in which an autonomous system for virtual private network, whose communicate with each other in a private area. For selecting a shortest path we focus on Steiner tree. The general concept of finding minimum path describes an undirected graph, in which vertices (node) and edges (links) are playing main role. we focus on Steiner tree problem, extra intermediate vertices and edges may be added to the graph in order to reduce the length of the spanning tree.
\end{abstract}

Keywords: Steiner tree, Autonomous system (AS).

\section{Introduction}

An Autonomous system for virtual private network means selected network established a path from source to destination is very challenging task. For getting a successful path from origin to destination Steiner tree is very helpful for choosing shortest path. Given a set $\mathrm{v}$ of points (vertices), interconnect them by a network (graph) of shortest length, where the length is the sum of the lengths of all edges.

The difference between the Steiner tree problem and the minimum spanning tree problem is that, in the Steiner tree problem, extra intermediate vertices and edges may be added to the graph in order to reduce the length of the spanning tree. These new vertices introduced to decrease the total length of connection are known as Steiner points or Steiner vertices, the resulting connection is a tree, known as the Steiner tree.

\section{Undirected Graph For Vpn}

Let $G=(\mathrm{V}, \mathrm{E}, \mathrm{c}, \mathrm{p})$ be an undirected graph, with the vertices $\mathrm{V}$ associated with non-negative profits $\mathrm{p}$, and with the edges $\mathrm{E}$ associated with non-negative costs $\mathrm{c}$. The graph may correspond to the vpn for local street map, for example, with the edges representing street segments and vertices representing street intersections and the location of potential customers. The profit $\mathrm{p}$ associated with a vertex is an estimate of the potential gain of revenue caused by that customer being connected to the network and receiving its service. Vertices corresponding to street intersections have profit zero. The cost $\mathrm{c}$ associated with an edge is the cost of establishing the connection, i.e., of laying the pipe or cable on the corresponding street segment.

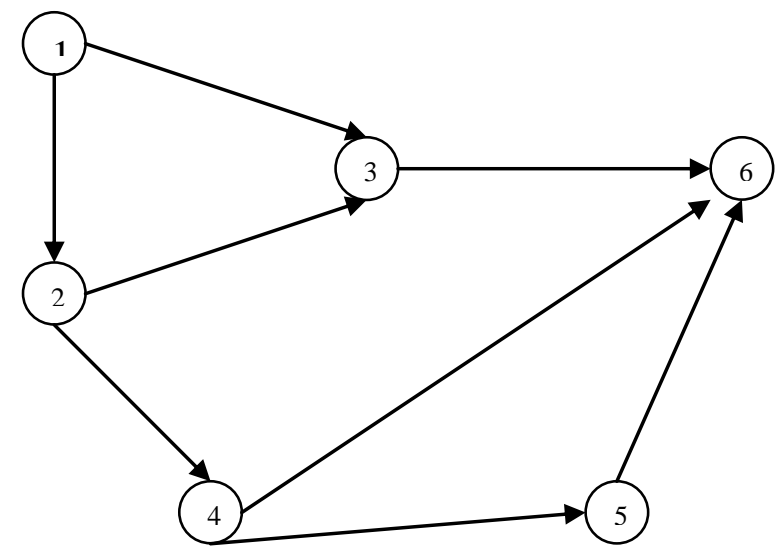

Fig-1 undirected graph for selected network

Nodes 1,2,3,4,5,6 are represent as vertices and show street interconnection and arrow represent as edges and show the street segment.

This undirected graph show selected network represent source that is node 1 and destination that is node 6 . Nodes 2,3,4,5 are intermediate nodes whose help to establish a path from source to destination ,this type of undirected graph make an autonomous system for private network. 


\subsection{Methodology}

An autonomous system for vpn creating selected network represent as graph in which finite nodes or links or we can say finite edges and vertices.

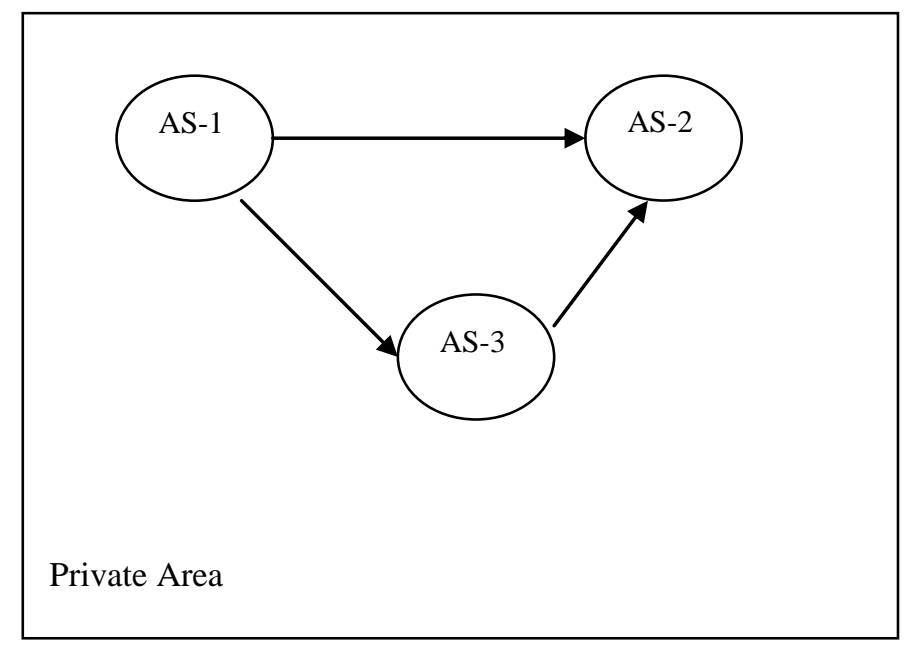

Fig-2, AS-1, AS-2 \& AS-3 are autonomous system and creating an private network, communicate with each other for establishing a path. Each autonomous system having undirected graph and these undirected graphs as the form of autonomous system communicate to each other in private area.

\subsection{Graph for AS-1 AS-2 and AS-3}

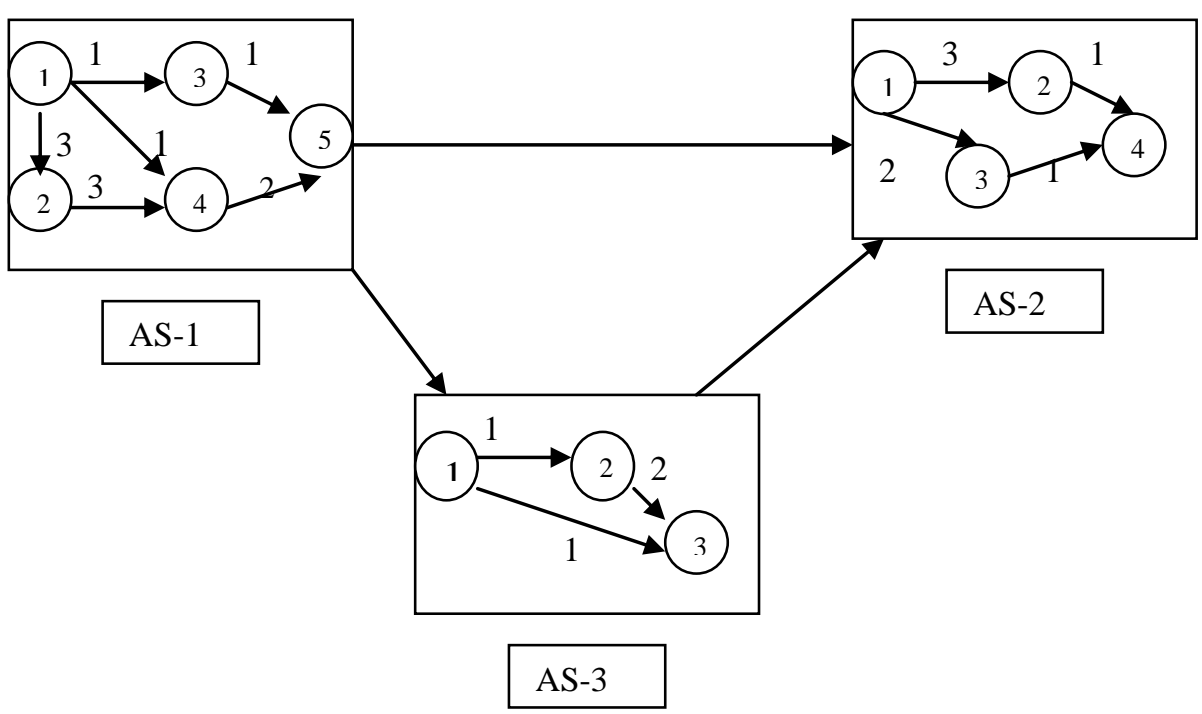

Fig-3, graph connected to each other in autonomous systems

In AS-1 node 1, 2,3,4,5 are connected to each other, there source is node 1 and destination is node 5 .for establishing path, each edges having some cost that is node 1 to node 3 having 1 , node 3 to 5 having cost 1 , node 1 to node 2 having cost 3 , node 2 to node 4 having cost 3 , node 1 to node 4 having cost 1 and node 4 to node 5 having cost 2.for selecting shortest path from source to destination we apply the concept of Steiner tree.

\subsubsection{Steiner tree: for selecting shortest path}

For selecting shortest path from source to destination in an selected network we apply steiner tree. Network represented as graph, split these graph in tree and will obtain steiner point and obtain shortest path based on lowest cost .

In AS-1(Autonomous Systems), undirected graph having nodes1,2,3,4,5 and established path like

Path from node $1-3$ cost is 1

From node $1-2$ cost is 3

From node $1-4$ cost is 1 
From node $2-4$ cost is 3

From node $3-5$ cost is 1

From node $4-5$ cost is 2

For AS-1

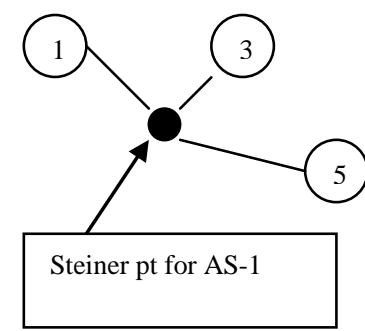

For AS-2

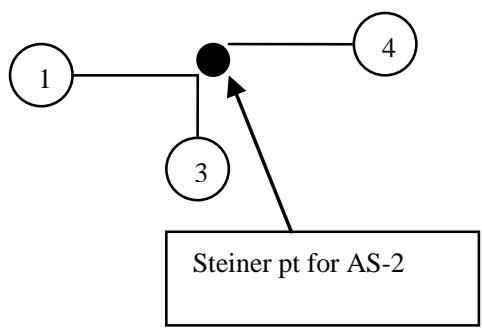

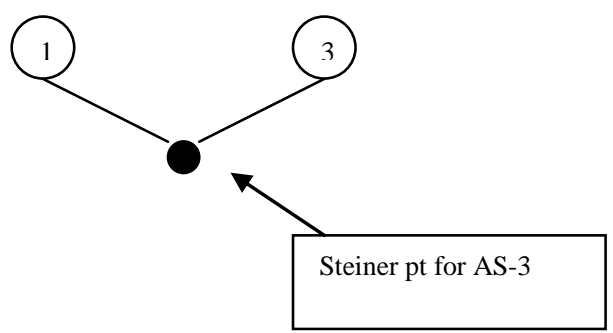

For As-3

Fig-4 showing the Steiner points

\section{Result}

It is an alternative approach for selecting shortest path from source to destination. This is a very useful algorithm, but if we are allowed a little bit more freedom in how we build the pipe network we can get a better solution. One obvious extension is to say, let us not restrict ourselves to only having links go between nodes directly, but allow the introduction of new points that the links can go through. If we allow these points to go anywhere and do not restrict the number of points, we can get better (lower cost) solutions. These new points are called Steiner points.

Finding Steiner points involves two problems, how many Steiner points should be used and where should they be placed.

\section{Conclusion}

In selected network choosing shortest path from source to destination find the least cost solution, a simple algorithm can be used. The algorithm starts by connecting the two closest nodes hen continues to find the shortest link that does not create a cycle and joining those nodes until all the nodes are connected. This is known as the minimum spanning tree algorithm or MST. But the Steiner tree reduce the no of interconnects between the nodes. reducing the overall bandwidth utilization.

\section{Acknowledgements}

I would like to thank Dr. Kanak Saxena (prof., Head, Department of Computer Applications) for her support, encouragement and guidance me for this paper.

\section{REFERENCES}

[1] D.-Z. Du, J. Smith and J. Rubinstein, Advances in Steiner Trees., Dordrecht: Kluwer Academic Publishers, 2000.

[2] G. Singh, S. Das, S. V. Gosavi and S. Pujar, "Ant Colony Algorithms for Steiner Trees: An Applicatoin to Routing in Sensor Networks," in Recent Developments in Biologically Inspired Computing, 2005, pp. 180-205.

[3] S. Das, S. Gosavi, W. Hsu and S. Vaze, "An Ant Colony Approach for the Steiner Tree Problem," New York, Proc. of Genectic and Evolutionay Computing Conference, 2012.

[4] L. Tay, 18 February 2011. [Online]. Available: http://www.itnews.com.au/News/248359, ants-buildcheapest- networks.aspx. [Accessed 11 August 2012]. 
[5] H. J. Promel and A. Steger. The Steiner Tree Problem: A Tour Through Graphs, Algo-rithms, and Complexity. Friedrich Vieweg and Son, Braunschweig, 2002.

[6] M. R. GAREY, R. L. GRAHAM and D. S. JOHNSON (1977) The complexity of computing Steiner minimal trees. SIAM J. AppI. Math. 34, 477-495. 32.

[7] J. HESSER, R. MANNER and 0. STUCKY (1989) Optimization of Steiner trees using genetic algorithms. Proc. 3rd Int. Conf. Genetic Algorithms (J. D. SCHAFFER, Ed.) 231-236.

[8] Glencora Borradaile, Claire Kenyon-Mathieu, Philip Klein, A polynomial-time approximation scheme for Steiner tree in planar graphs, Proceedings of the eighteenth annual ACM-SIAM symposium on Discrete algorithms, p.1285-1294, January 07-09, 2007, New Orleans, Louisiana

[9] S. E. Dreyfus and R. A. Wagner. The Steiner problem in graphs. Networks, 1:195--207, 1972.

[10] Deeparnab Chakrabarty, Nikhil R. Devanur, Vijay V. Vazirani, New geometry-inspired relaxations and algorithms for the metric steiner tree problem, Proceedings of the 13th international conference on Integer programming and combinatorial optimization, May 26-28, 2008, Bertinoro, Italy 